\title{
EACH LOCALLY ONE-TO-ONE MAP FROM A CONTINUUM ONTO A TREE-LIKE CONTINUUM IS A HOMEOMORPHISM
}

\author{
JO W. HEATH
}

(Communicated by James West)

\begin{abstract}
In 1977 T. Maćkowiak proved that each local homeomorphism from a continuum onto a tree-like continuum is a homeomorphism. Recently, J. Rogers proved that each locally one-to-one (not necessarily open) map from a hereditarily decomposable continuum onto a tree-like continuum is a homeomorphism, and this paper removes "hereditarily decomposable" from the hypothesis of Rogers' theorem.
\end{abstract}

It is not easy for a nice function to map onto a tree-like continuum without being a homeomorphism. T. Maćkowiak's classic result [3], proved in 1977, is that each local homeomorphism from a continuum onto a tree-like continuum is a homeomorphism. Local homeomorphisms are both open and locally one-to-one, and recently J. Rogers asked if "open" could be removed from the hypothesis of the Maćkowiak theorem. In [1] Rogers proved a special case, namely that if a locally one-to-one map that is not a homeomorphism is defined on a hereditarily decomposable continuum, then the image contains a continuum that is not unicoherent. Since all tree-like continua are hereditarily unicoherent, the image cannot be tree-like. These results come from the "complicated proof" found in [2] of Corollary 5.7 in [1]. We use the noun "map" to mean continuous function, and the term "continuum" to mean a connected, compact metric space.

The theorem to follow completes the task of removing "open" from the Mackowiak theorem. The lemma that is proved first helps to organize the covers.

Definition. A finite collection of sets has a tree-indexing if its members can be labeled $\left\{L_{1}, L_{2}, \ldots, L_{m}\right\}$ so that the $L_{i}$ are distinct and for each $j$ from 2 to $m, L_{j}$ intersects exactly one member of the set $\left\{L_{1}, L_{2}, \ldots, L_{j-1}\right\}$.

Tree-cover lemma. A finite collection of open sets has a tree-indexing iff its nerve is a tree.

Proof. Suppose $\left\{L_{1}, L_{2}, \ldots, L_{m}\right\}$ is a tree-indexing of a finite collection $\mathcal{V}$ of open sets. Then $\mathcal{V}$ is coherent since if $\mathcal{V}=\mathcal{V}_{1} \cup \mathcal{V}_{2}$, two disjoint subcollections whose unions do not intersect, and if $L_{1} \in \mathcal{V}_{1}$, then the element of $\mathcal{V}_{2}$ with the smallest label fails the definition of a tree-indexing. Since $\mathcal{V}$ is coherent, its nerve is connected. Since no three elements in $\mathcal{V}$ can intersect, its nerve is a one-dimensional graph. If the nerve of $\mathcal{V}$ contains a simple closed curve, then the element of $\mathcal{V}$ with the

Received by the editors January 30, 1995.

1991 Mathematics Subject Classification. Primary 54C10.

Key words and phrases. Tree-like, locally one-to-one, chain, tree-indexing, continuum.

(C)1996 American Mathematical Society 
largest label, that corresponds to some vertex in the simple closed curve, violates the tree-indexing definition. Therefore the nerve of $\mathcal{V}$ is a tree. Now, by way of contradiction, let $\mathcal{V}$ denote the smallest finite collection of open sets whose nerve is a tree but does not have a tree-indexing. Since the nerve of $\mathcal{V}$ is connected, the collection $\mathcal{V}$ must be coherent. Clearly, $\mathcal{V}$ must have more than two elements. Since the nerve of $\mathcal{V}$ has at least three vertices, the removal of an endpoint $e$, and the arc that connects $e$ to the rest of the tree, leaves a tree. By our assumption, this means that the collection $\mathcal{V} \backslash\{V(e)\}$, where $V(e)$ denotes the open set in $\mathcal{V}$ that corresponds to $e$, has a tree-indexing $\left\{L_{1}, L_{2}, \ldots, L_{m}\right\}$. This generates a tree-indexing for $\mathcal{V}$ by labeling $V(e)$ as $L_{m+1}$.

Theorem. Every locally one-to-one map from a continuum onto a tree-like continuum is a homeomorphism.

Proof. Suppose that $h$ is a locally one-to-one map from a continuum $X$ onto a treelike continuum $Y$. It is clear that $h$ must be finite-to-one, and there is a positive number $\epsilon$ such that if $x$ and $x^{\prime}$ are points of $X$ such that $h(x)=h\left(x^{\prime}\right)$, then $d\left(x, x^{\prime}\right)>3 \epsilon$. For each point $y$ in $Y$, there is an open set $U(y)$ in $Y$ containing $y$ such that if $h^{-1}(y)=\left\{x_{1}, x_{2}, \ldots, x_{n}\right\}$, then $h^{-1}(U(y)) \subseteq \bigcup_{i=1}^{n} N_{\epsilon}\left(x_{i}\right)$ in $X$, where $N_{\epsilon}\left(x_{i}\right)$ denotes the $\epsilon$ neighborhood of $x_{i}$ in $X$. Now, let $\mathcal{V}$ denote an open refinement of $\{U(y) \mid y \in Y\}$ that covers $Y$ and whose nerve is a tree. By the tree-cover lemma, $\mathcal{V}$ can be written $\left\{L_{1}, L_{2}, \ldots, L_{m}\right\}$, satisfying the definition of a tree-indexing. For each $L_{i} \in \mathcal{V}$, let $y_{i}$ denote a member of $Y$ such that $L_{i} \subseteq U\left(y_{i}\right)$. Index the elements of $h^{-1}\left(y_{i}\right)=\left\{x_{1}, x_{2}, \ldots, x_{k}\right\}$, and for $j=1, \ldots, k$, define $W(i, j)=h^{-1}\left(L_{i}\right) \cap N_{\epsilon}\left(x_{j}\right)$, if this set is non-empty. Note that for each relevant $i$ and $j, W(i, j)$ is an open set in $X$ of diameter less than $\epsilon$, and $h$ is one-to-one on $W(i, j)$. Now define $\mathcal{W}$ to be the set of these $W(i, j)$ 's. Then $\mathcal{W}$ is an open covering of $X$.

3-link fact. If $W\left(i_{1}, j_{1}\right), W\left(i_{2}, j_{2}\right)$, and $W\left(i_{3}, j_{3}\right)$ are distinct elements of $\mathcal{W}$ such that $W\left(i_{2}, j_{2}\right)$ intersects each of the other two, then the integers $\left\{i_{1}, i_{2}, i_{3}\right\}$ are distinct.

Let $z_{1}$ denote a point of $W\left(i_{1}, j_{1}\right) \cap W\left(i_{2}, j_{2}\right)$, let $z_{3}$ denote a point of $W\left(i_{3}, j_{3}\right) \cap$ $W\left(i_{2}, j_{2}\right)$, and note that $d\left(z_{1}, z_{3}\right)<\epsilon$. First, suppose that $i_{1}=i_{2}$. By construction, $W\left(i_{1}, j_{1}\right) \subseteq N_{\epsilon}\left(x_{j_{1}}\right)$ and $W\left(i_{1}, j_{2}\right) \subseteq N_{\epsilon}\left(x_{j_{2}}\right)$, where $h\left(x_{j_{1}}\right)=h\left(x_{j_{2}}\right)=y_{i_{1}}$. But $z_{1} \in N_{\epsilon}\left(x_{j_{1}}\right) \cap N_{\epsilon}\left(x_{j_{2}}\right)$ implies that $d\left(x_{j_{1}}, x_{j_{2}}\right)<2 \epsilon$, that is, $j_{1}=j_{2}$. This is contrary to the fact that the $W$ 's are distinct. A similar contradiction occurs if $i_{3}=i_{2}$. Secondly, suppose that $i_{1}=i_{3}$. Again, by construction, $z_{1} \in W\left(i_{1}, j_{1}\right) \subseteq N_{\epsilon}\left(x_{j_{1}}\right)$ and $z_{3} \in W\left(i_{1}, j_{3}\right) \subseteq N_{\epsilon}\left(x_{j_{3}}\right)$, where $h\left(x_{j_{1}}\right)=h\left(x_{j_{3}}\right)=y_{i_{1}}$. Thus $d\left(x_{j_{1}}, x_{j_{3}}\right)>3 \epsilon$ if $j_{1} \neq j_{3}$. But this is contrary to the fact that each of the following numbers is less than $\epsilon: d\left(x_{j_{1}}, z_{1}\right), d\left(z_{1}, z_{3}\right)$, and $d\left(x_{j_{3}}, z_{3}\right)$. This contradiction completes the proof of the 3 -link fact.

Now, back to the proof of the theorem. If $h$ is not a homeomorphism, then $h$ is not one-to-one, so there exist two points $x_{1}$ and $x_{2}$ such that $h\left(x_{1}\right)=h\left(x_{2}\right)$. So $x_{1} \in$ $W(i, j)$ and $x_{2} \in W(i, k)$ for some $i$ and $j \neq k$, and there is a chain of elements from $\mathcal{W}$ with first link $W(i, j)$ and last link $W(i, k)$. Let $C=\left\{W\left(k_{1}, n_{1}\right), W\left(k_{2}, n_{2}\right), \ldots\right.$, $\left.W\left(k_{m}, n_{m}\right)\right\}$ denote a chain in $\mathcal{W}$ of shortest length such that $k_{1}=k_{m}$. By the 3-link fact, $m>3$. The indexing on $C$ is understood to be the usual chain indexing, where the links are distinct and $W\left(k_{i}, n_{i}\right)$ intersects $W\left(k_{j}, n_{j}\right)$ iff $|i-j| \leq 1$. Let $k_{j}$ be the smallest integer in $\left\{k_{1}, k_{2}, \ldots, k_{m}\right\}$, where we use $j=1$ if the smallest is $k_{1}=k_{m}$. Then $k_{j+1}>k_{j}$ and $k_{j+2}>k_{j+1}$. To see this second inequality, note 
that in $\mathcal{V}, L_{k_{j+1}}$ intersects $L_{k_{j}}$ since $W\left(k_{j+1}, n_{j+1}\right)$ intersects $W\left(k_{j}, n_{j}\right)$, so $L_{k_{j}}$ is the only element of $\mathcal{V}$ with lower subscript that $L_{k_{j+1}}$ intersects. This means that, since $L_{k_{j+1}}$ also intersects $L_{k_{j+2}}$, the subscript $k_{j+2}$ must be greater than $k_{j+1}$. If we continue in this way we can establish the fact that $k_{j}<k_{j+1}<k_{j+2}<\ldots<$ $k_{m}=k_{1}<k_{2}<\ldots<k_{j-1}$. Note that when we "turn the corner" we use the fact that $k_{2} \neq k_{m-1}$, which follows since $m>3$. The final contradiction is that the last link $L_{k_{j-1}}$ intersects both of the lower indexed links $L_{k_{j-2}}$ and $L_{k_{j}}$.

\section{REFERENCES}

[1] James T. Rogers, Jr., Diophantine conditions imply critical points on the boundaries of Siegel disks of polynomials I, Preprint.

[2] James T. Rogers, Jr., Critical points on the boundaries of Siegel disks, Bull. Amer. Math. Soc. (N.S.) 32 (1995), 317-321. MR 96a:30032

[3] T. Maćkowiak, Local homeomorphisms onto tree-like continua, Colloq. Math. XXXVIII (1977), 63-68. MR 57:4135

Department of Mathematics, Auburn University, Alabama 36849-5310

E-mail address: heathjw@mail.auburn.edu 